\title{
FULL COUNTING STATISTICS IN ELECTRIC CIRCUITS
}

\author{
MARKUS KINDERMANN \\ Instituut-Lorentz, Universiteit Leiden, P.O. Box 9506, 2300 \\ $R A$ Leiden, The Netherlands \\ AND \\ YULI V. NAZAROV \\ Department of Nanoscience, Delft University of Technology, \\ Lorentzweg 1, 2628 CJ Delft, The Netherlands
}

\section{Introduction}

Full counting statistics (FCS) is one of the most attractive and intellectually involved concepts in quantum transport. It provides much information about charge transfer (all moments, including average current, noise, etc.) in a compact and elegant form. Albeit the study of full counting statistics has begun with some confusion. The first attempt to derive FCS [1] exploited the text-book definition of quantum measurement: the probability of the outcome $q$ of a measurement is given by $\langle n|\delta(q-\hat{Q})| n\rangle, \hat{Q}$ being an operator associated with the value measured and $|n\rangle$ the quantum state. However, the choice of $Q$ made in [1] resulted in severe interpretation problems. In [2] the authors revised their approach. The new method, that is commonly accepted now, invokes an extra degree of freedom, a detector. The quantum measurement paradigm is applied to the detector degree of freedom.

There is a similarity between the method of [2] and two core approaches to quantum dynamics: the Keldysh technique [3] and the Feynman-Vernon formalism [4]. This similarity was not stressed in [2]. Later it has been noticed that the FCS can be evaluated with a straightforward modification of the Keldysh Green function technique [5]. This allows one to extend the studies of the FCS to many systems, some results being reviewed in other contributions to this book. This is good news. Seemingly bad news is that the method of [2] does not always give results that can be interpreted as probabilities of measurement outcomes. In the statistics of charge transfer, 
the bad news becomes relevant for systems where gauge invariance is broken $[6,7]$. If one tries to generalize FCS to arbitrary variables, these problems arise from the very beginning [8].

The present contribution consists of two parts. First we address the bad news. For this, we consider a rather general and abstract problem, the counting statistics of a general quantum mechanical variable being measured by a linear detector. We find that in this case the FCS is physically meaningful and useful since it determines the quantum time evolution of the detector. This is despite the fact that it can not be interpreted as a probability distribution [8].

Thus encouraged, we investigate how far one can go with this approach. The abstract exercise with a linear detector proves to be very useful to describe a mesoscopic conductor embedded in a linear electric circuit. We reveal the relations between the FCS and the non-equilibrium Keldysh action that describes current and voltage fluctuations in electric circuits. We show how to evaluate the current and voltage counting statistics at any two contacts of an arbitrary circuit. This appears to be relevant for future experimental activities in the field of quantum noise and statistics [9].

The article is organized as follows. In section 2 we will pursue a route similar to that proposed in [1], defining a current statistics as the statistics of a charge operator. This route has not been explored before. It allows to understand the origin of the "negative probabilities" found in [6] purely in terms of conductor degrees of freedom. We follow an alternative route in section 3 and consider a system coupled to a static linear detector [8]. We show that the result of the detection depends in general on the initial state of the detector. To predict the result, one needs a function of two variables. We adopt then this function as the definition of the full counting statistics. We will identify situations where the two approaches give the same easy-to-interpret result.

Then we turn to electric circuits. We explain in section 4 why a linear circuit can be viewed as a set of dynamical linear detectors and why the FCS expression is the building block of the Keldysh action that describes the quantum dynamics of the circuit. In section 5 we discuss the low-frequency limit of the action. In this limit, the action is local in time. This facilitates its evaluation. The general scheme is illustrated in section 6 with a simple model of a phase-coherent conductor in series with an external linear resistor. In this case, we are able to determine the full statistics of current and voltage fluctuations. These results were obtained in collaboration with C.W.J. Beenakker, that we gladly acknowledge. 


\section{Charge statistics without detector}

We start out by defining a statistics of time averages of electric current without specifying a measurement procedure, as it has been also done in [7]. Unlike in [7] we study directly the statistics of charge $Q$ on one side of the cross-section of a conductor through which the current is measured. If $Q$ is fixed at time 0 , the statistics of $Q(\tau)$ at time $\tau$ corresponds to the statistics of the charge that has traversed the cross-section in the time interval $[0, \tau]$. Having the clear physical interpretation of the statistics of charge on one side of the cross-section, this definition will shed some light on interpretation problems encountered with other definitions, like "negative probabilities" and half-integer charge transfer [6, 7].

To specify $Q$ we introduce, following [2], a smooth function $f$ that divides the conductor into two parts: a left side, where $f(\mathbf{x})<0$, and a right side, where $f(\mathbf{x})>0$. We are interested in charge transfer through the boundary $f(\mathbf{x})=0$ between these two sides. The operator of electric charge to the right of the cross-section is $Q=e \theta[f(\mathbf{x})]$ [where $\theta(x)=0$ for $x \leq 0$ and $\theta(x)=1$ for $x>0$ ], $e$ being the elementary charge. It is convenient to express the statistics of $Q(\tau)$ via the corresponding moment generating function

$$
\chi_{\mathrm{c}}(\lambda)=\sum_{k} \frac{(i \lambda)^{k}}{k !}\left\langle Q(\tau)^{k}\right\rangle=\left\langle e^{i \lambda Q(\tau)}\right\rangle=\left\langle e^{i H \tau} e^{i \lambda Q} e^{-i H \tau}\right\rangle .
$$

Here $H$ is the Hamiltonian of the current conductor and the average is taken over the initial density matrix of the conductor.

One advantage of defining $\chi_{\mathrm{c}}$ as in Eq. (1) is that it is evidently associated with a (positive) probability distribution, the distribution of measurement outcomes of an observable corresponding to the Hermitian operator $Q(\tau)$. Besides, $\chi_{\mathrm{c}}$ predicts charge transfer in integer multiples of the electron charge $e$ for systems of well-localized non-interacting electrons, the result to be expected. Both very physical properties have been found to be violated by other definitions $[6,7]$. One buys these advantages by defining a statistics that is only indirectly linked to the statistics of transmitted charge. That link requires the knowledge of the initial charge state of the conductor.

Using that

$$
e^{i \lambda Q / 2} e^{A} e^{-i \lambda Q / 2}=\exp \left[e^{i \lambda Q / 2} A e^{-i \lambda Q / 2}\right]
$$

we rewrite Eq. (1) identically as

$$
\begin{aligned}
\chi_{\mathrm{c}}(\lambda) & =\left\langle e^{i \lambda Q / 2} \exp \left[i e^{-i \lambda Q / 2} H e^{i \lambda Q / 2} \tau\right]\right. \\
& \left.\exp \left[-i e^{i \lambda Q / 2} H e^{-i \lambda Q / 2} \tau\right] e^{i \lambda Q / 2}\right\rangle .
\end{aligned}
$$


The charge operator $Q$ commutes with all position operators $\mathbf{x}$ contained in the Hamiltonian $H=H(\mathbf{p}, \mathbf{x})$. It does, however, not commute with the momentum operators $\mathbf{p}$. As a consequence, momentum operators are transformed as

$$
e^{-i \lambda Q / 2} \mathbf{p} e^{i \lambda Q / 2}=\mathbf{p}-\frac{e}{2} \lambda \nabla \theta[f(\mathbf{x})] \equiv \tilde{\mathbf{p}}_{\lambda} .
$$

We define a new Hamiltonian $H_{\lambda}=H\left(\tilde{\mathbf{p}}_{\lambda}, \mathbf{x}\right)$ in the same way as it has been done in [2]. The generating function

$$
\chi_{\mathrm{c}}(\lambda)=\left\langle e^{i \lambda Q / 2} e^{i H_{-\lambda} \tau} e^{-i H_{\lambda} \tau} e^{i \lambda Q / 2}\right\rangle
$$

takes then a form that is very similar to the $\chi(\lambda)$ found in [2] within the spin- $\frac{1}{2}$ current detection model . Tracing back the difference we write $\chi(\lambda)$ in terms of charge operators as

$$
\chi(\lambda)=\left\langle e^{-i \lambda Q / 2} e^{i \lambda Q(\tau)} e^{-i \lambda Q / 2}\right\rangle .
$$

If the initial state of the conductor is an eigenstate of charge with eigenvalue $Q_{0}$, the two generating functions are identical up to the offset charge $Q_{0}, \chi(\lambda)=e^{-i \lambda Q_{0}} \chi_{\mathrm{c}}(\lambda)$. In this case both characteristic functions are associated with a positive probability

$$
P(q)=\int d \lambda e^{-i q \lambda} \chi(\lambda)
$$

to transfer $q$ charges during the measurement time. For generic systems of non-interacting electrons this probability has been found to be nonzero only at integer multiples of the electron charge, corresponding to the transfer of an integral number of electrons [2].

If the initial state is a superposition of eigenstates of charge $Q$, the two generating functions $\chi$ and $\chi_{\mathrm{c}}$ differ. For example, $\chi$ may seem to predict the transfer of half the elementary charge for systems of well-localized non-interacting electrons, when $\chi_{\mathrm{c}}$ indicates integer charge transfer. This becomes evident when $\chi$ is written in the form (6). It contains summands of the form $\chi_{m n}=\exp [-i \lambda(m+n) / 2]\langle m, \alpha|\exp [i \lambda Q(\tau)]| n, \beta\rangle,|m, \alpha\rangle$ and $|n, \beta\rangle$ being eigenstates of the charge with additional quantum numbers $\alpha$, $\beta, Q|m, \alpha\rangle=m|m, \alpha\rangle$. While the matrix element in the expression for $\chi_{m n}$ corresponds to integer charge transfer, the pre-factor suggests the transfer of half-integral charges when $m+n$ is an odd number. This has been observed in [7].

For a Josephson junction at the cross-section $f(\mathbf{x})=0$ between two superconductors the situation is even more involved. One would like to choose 
an initial state of well defined phase difference between the two sides of the junction in order to have a well defined current flowing. However, phase and charge are conjugated variables obeying Heisenberg's uncertainty principle and the dispersion of the initial charge $Q$ in such a state is infinite. The charge distribution corresponding to $\chi_{\mathrm{c}}$ with an initial state of well-defined phase is therefore one of undetermined $Q(\tau)$. It contains no information about the charge transfer. This is clearly undesirable. Because all the uncertainty of $Q$ is already present in the initial state and has nothing to do with the transfer process, one might hope to be able to eliminate it in a meaningful way. Indeed, we have seen that this is accomplished for the initial charge offset in a state of well defined charge by the factors $e^{-i \lambda Q / 2}$ in the definition of $\chi(\lambda)(6)$. One could hope that this works also for the initial charge spread in a phase eigenstate. Instead, $\chi(\lambda)$ then seems to imply "negative probabilities" [6].

A way to remedy this problem would be to calculate $\chi_{\mathrm{c}}$ in an initial state with a finite charge and phase dispersion. One could also try to find general relations between the charge statistics and the initial state of the conductor that would characterize the charge transfer process more generally.

The alternative is to couple the (super-)conductor to a detector and interpret the detector read-off in terms of charge transfer. We will follow this route in the next section. It turns out that in a idealized detector model a function very similar to the generating function $\chi$ eventually determines the final state of the detector provided the initial state is known. The charge transfer through the conductor is then characterized by its effect on the detector.

\section{Counting statistics with a static detector}

We turn now to the measurement process of time averaged quantities. In this section we focus on an idealized measuring device without its own dynamics, a static detector. Within this model we study the measurement of time averages $\int_{0}^{\tau} d t A(t)$ of an arbitrary operator $A$. For electric currents, $A=I$, this will allow us to characterize the charge transfer and its statistics by a function similar to the characteristic function introduced in the previous section. The analysis of this function will allow us to trace the origin of the "negative probabilities" found in [6].

\subsection{DETECTOR MODEL}

We employ a detector model that has first been used by John von Neumann in an analysis of the quantum measurement process. Following [10] e introduce a detector variable whose operator $x$ commutes with all operators of the system to be measured. Its Hamiltonian is $q^{2} / 2 m$, where $q$ is 
the variable conjugated to $x$. The system measured shall be coupled to the position $x$ of the detector during the time interval $[0, \tau]$ and be decoupled adiabatically for earlier and later times. For this we introduce a smooth coupling function $\alpha_{\tau}(t)$ that takes the value 1 in the time interval $[0, \tau]$ and is zero beyond the interval $\left[t_{1}, t_{2}\right]\left(t_{1}<0\right.$ and $\left.t_{2}>\tau\right)$. The values for $t_{1}<t<0$ and $\tau<t<t_{2}$ are chosen in a way that provides an adiabatic switching. The entire Hamiltonian reads then

$$
H(t)=H_{\mathrm{sys}}-\alpha_{\tau}(t) x A+\frac{q^{2}}{2 m} .
$$

The Heisenberg equation of motion for the detector momentum $q$

$$
\dot{q}(t)=\alpha_{\tau}(t) A(t)
$$

suggests, that the statistics of outcomes of measurements of the detector's momentum after having it uncoupled from the system corresponds to the statistics of the time average $\int_{0}^{\tau} d t A(t)$ that we are interested in.

The coupling term can be viewed as a disturbance of the system measured by the detector. To minimize this disturbance, we would clearly like to concentrate the detector wave function around $x=0$. The uncertainty principle forbids us, however, to localize it completely. Thereby we would loose all information about the detector momentum, which we intend to measure. This is a fundamental limitation imposed by quantum mechanics, and we are going to explore its consequences step by step. To discern it form a classical back action of the detector we take the limit of a static detector with $m \rightarrow \infty$, such that $\dot{x}=0$ and any classical back action is ruled out.

\subsection{APPROACH}

To predict the statistics of measurement outcomes in our detection model we need the reduced density matrix of the detector after the measurement, at $t>t_{2}$. If there were no system to measure we could readily express it in the form of a path integral in the (double) variable $x(t)$ over the exponential of the detector action. This is still possible in the presence of a system coupled to the detector [4]. The information about the system to be measured can be compressed into an extra factor in this path integral, the so-called influence functional. This makes the separation between the detector and the measured system explicit. To make contact with [2], we write the influence functional as an operator expression that involves system degrees of freedom only. We denote the initial detector density matrix (at $\left.t<t_{1}\right)$ by $\rho^{i n}\left(x^{+}, x^{-}\right)$and the final one (at $t>t_{2}$, after having traced out the system's degrees of freedom) by $\rho^{f}\left(x^{+}, x^{-}\right) . R$ denotes the initial 
density matrix of the system. The entire initial density matrix $D$ is assumed to factorize, $D=R \rho^{i n}$.

We start out by inserting complete sets of states into the expression for the time development of the density matrix

$$
\begin{gathered}
\rho^{f}\left(x^{+}, x^{-}\right)=\underset{\text { System }}{\operatorname{Tr}}\left\langle x^{+}\right| \vec{T} e^{-i \int_{t_{1}}^{t_{2}} d t\left[H_{\mathrm{sys}}-\alpha_{\tau}(t) x A+\frac{q^{2}}{2 m}\right]} D \\
\overleftarrow{T} e^{i \int_{t_{1}}^{t_{2}} d t\left[H_{\mathrm{sys}}-\alpha_{\tau}(t) x A+\frac{q^{2}}{2 m}\right]}\left|x^{-}\right\rangle
\end{gathered}
$$

Here, $\vec{T}(\overleftarrow{T})$ denotes (inverse) time ordering. As the complete sets of states we choose product states of any complete set of states of the system and alternatingly complete sets of eigenstates of the position or the momentum operator of the detector. Those intermediate states allow us to replace the position and momentum operators in the time development exponentials by their eigenvalues. We can then do the integrals over the system states as well as the momentum integrals and arrive at the expression

$$
\begin{aligned}
& \rho^{f}\left(x^{+}, x^{-}\right)=\int_{\substack{\mathcal{D}\left[x^{+}\right] \\
x^{+}\left(t_{2}\right)=x^{+} x^{-}\left(t_{2}\right)=x^{-}}}^{\mathcal{D}\left[x^{-}\right]} \rho^{i n}\left[x^{+}\left(t_{1}\right), x^{-}\left(t_{1}\right)\right] e^{-i \mathcal{S}_{\text {Det }}\left(\left[x^{+}\right],\left[x^{-}\right]\right)} \\
& \underset{\text { System }}{\operatorname{Tr}} \vec{T} e^{-i \int_{t_{1}}^{t_{2}} d t\left[H_{\mathrm{sys}}-\alpha_{\tau}(t) x^{+}(t) A\right]} R \overleftarrow{T} e^{i \int_{t_{1}}^{t_{2}} d t\left[H_{\mathrm{sys}}-\alpha_{\tau}(t) x^{-}(t) A\right]}
\end{aligned}
$$

with the detector action

$$
\mathcal{S}_{\text {Det }}\left(\left[x^{+}\right],\left[x^{-}\right]\right)=-\int_{t_{1}}^{t_{2}} d t \frac{m}{2}\left[\left(\dot{x}^{+}\right)^{2}-\left(\dot{x}^{-}\right)^{2}\right] .
$$

We rewrite the expression as

$$
\rho^{f}\left(x^{+}, x^{-}\right)=\int d x_{1}^{+} d x_{1}^{-} K\left(x^{+}, x^{-} ; x_{1}^{+}, x_{1}^{-}, \tau\right) \rho^{i n}\left(x_{1}^{+}, x_{1}^{-}\right)
$$

with the kernel

$$
\begin{aligned}
& K\left(x^{+}, x^{-} ; x_{1}^{+}, x_{1}^{-}, \tau\right)=\int_{x^{+}\left(t_{2}\right)=x^{+}, x^{+}\left(t_{1}\right)=x_{1}^{+}} \mathcal{D}\left[x^{+}\left(t_{2}\right)=x^{-}, x^{-}\left(t_{1}\right)=x_{1}^{-}\right. \\
& \mathcal{Z}_{\text {Sys }}\left(\left[\alpha_{\tau} x^{+}\right],\left[\alpha_{\tau} x^{-}\right]\right) e^{-i \mathcal{S}_{\text {Det }}\left(\left[x^{+}\right],\left[x^{-}\right]\right)}
\end{aligned}
$$

that contains the influence functional

$$
\mathcal{Z}_{\text {Sys }}\left(\left[\chi^{+}\right],\left[\chi^{-}\right]\right)=\underset{\text { System }}{\operatorname{Tr}} \vec{T} e^{-i \int_{t_{1}}^{t_{2}} d t\left[H_{\mathrm{sys}}-\chi^{+}(t) A\right]} R \overleftarrow{T} e^{i \int_{t_{1}}^{t_{2}} d t\left[H_{\mathrm{sys}}-\chi^{-}(t) A\right]}
$$


Taking the limit of an infinite detector mass now, we find that $\mathcal{S}_{\text {Det }}$ in Eq. (14) suppresses all fluctuations in the path integral. Therefore, the kernel $K\left(x^{+}, x^{-}, x_{1}^{+}, x_{1}^{-}, \tau\right)$ becomes local in position space,

$$
K\left(x^{+}, x^{-}, x_{1}^{+}, x_{1}^{-}, \tau\right)=\delta\left(x^{+}-x_{1}^{+}\right) \delta\left(x^{-}-x_{1}^{-}\right) P\left(x^{+}, x^{-}, \tau\right)
$$

with

$$
P\left(x^{+}, x^{-}, \tau\right)=\underset{\text { System }}{\operatorname{Tr}} \vec{T} e^{-i \int_{t_{1}}^{t_{2}} d t\left[H_{\mathrm{sys}}-\alpha_{\tau}(t) x^{+} A\right]} R \overleftarrow{T} e^{i \int_{t_{1}}^{t_{2}} d t\left[H_{\mathrm{sys}}-\alpha_{\tau}(t) x^{-} A\right]}
$$

It is constructive to rewrite now the density matrices in the Wigner representation

$$
\rho(x, q)=\int \frac{d z}{2 \pi} e^{-i q z} \rho\left(x+\frac{z}{2}, x-\frac{z}{2}\right)
$$

and define

$$
P(x, q, \tau)=\int \frac{d z}{2 \pi} e^{-i q z} P\left(x+\frac{z}{2}, x-\frac{z}{2}, \tau\right) .
$$

With these definitions we find a compact relation that summarizes the results of this subsection:

$$
\rho^{f}(x, q)=\int d q_{1} P\left(x, q-q_{1}, \tau\right) \rho^{i n}\left(x, q_{1}\right) .
$$

\subsection{INTERPRETATION OF THE FCS}

We adopt the relations (17), (19) and (20) as the definition of the FCS of the variable $A$. Let us see why. First let us suppose that we can treat the detector classically. Then the density matrix of the detector in the Wigner representation can be interpreted as a classical probability distribution $\Pi(x, q)$ to be at a certain position $x$ with momentum $q$. This allows for a classical interpretation of $P(x, q, \tau)$ as the probability to have measured $q=\int_{0}^{\tau} A(t)$. Indeed, one sees from $(20)$ that $\rho^{f}(x, q)$ is obtained from $\rho^{\text {in }}$ by shifts in $q, P(x, q, \tau)$ being the probability distribution of those shifts.

In general, the density matrix in the Wigner representation cannot be interpreted as a probability to have a certain position and momentum since it is not positive. Concrete calculations given below illustrate that $P(x, q, \tau)$ does not have to be positive either. Consequently, it cannot be interpreted as a probability distribution. Still it predicts the results of measurements according to Eq. (20). 
There is, however, an important case when the FCS can indeed be interpreted as a probability distribution. It is the case that $P(x, q, \tau)$ does not depend on $x, P(x, q, \tau) \equiv P(q, \tau)$. Then, integrating Eq. (20) over $x$, we find

$$
\Pi^{f}(q)=\int d q^{\prime} P\left(q-q^{\prime}, \tau\right) \Pi^{i n}\left(q^{\prime}\right)
$$

with $\Pi(q) \equiv \int d x \rho(x, q)$. Therefore, the FCS is in this special case the kernel that relates the probability distributions of the detector momentum before and after the measurement, $\Pi^{i n}(q)$ and $\Pi^{f}(q)$, to each other. Those distributions are positive and so is $P(q, \tau)$.

When studying the FCS of a stationary system and the measurement time $\tau$ exceeds time scales associated with the system, the operator expression in Eq. (17) can be seen as a product of terms corresponding to time intervals. Therefore in this limit of $\tau \rightarrow \infty$ the dependence on the measuring time can be reconciled into

$$
P\left(x^{+}, x^{-}, \tau\right)=e^{-\mathcal{E}\left(x^{+}, x^{-}\right) \tau}
$$

where the expression in the exponent is supposed to be large. Then the integral (19) that defines the FCS can be done in the saddle point approximation. Defining the time average $\bar{A}=q / \tau$, that is, $\bar{A}$ is the result of a measurement of $\int_{0}^{\tau} A(t) d t / \tau$, the FCS can be recast into the form

$$
P(x, \bar{A}, \tau)=e^{-\tilde{\mathcal{E}}(x, \bar{A}) \tau},
$$

where $\tilde{\mathcal{E}}$ is defined as the (complex) extremum with respect to (complex) $z$ :

$$
\tilde{\mathcal{E}}=\operatorname{extr}_{z}\left\{\mathcal{E}\left(x+\frac{z}{2}, x-\frac{z}{2}\right)+i \bar{A} z\right\} .
$$

The average value of $\bar{A}$ and its variance (noise) can be expressed in terms of derivatives of $\mathcal{E}$ :

$$
\begin{aligned}
\langle\bar{A}\rangle & =-\lim _{z \rightarrow 0} \frac{\partial \mathcal{E}(x+z / 2, x-z / 2)}{i \partial z} \\
\tau\left\langle\left\langle\bar{A}^{2}\right\rangle\right\rangle & =\lim _{z \rightarrow 0} \frac{\partial^{2} \mathcal{E}(x+z / 2, x-z / 2)}{\partial z^{2}} .
\end{aligned}
$$

More generally, the quantity $P\left(x^{+}, x^{-}, \tau\right)$ is the generating function of moments of $q$. It is interesting to note that in general this function may generate a variety of moments that differ in the time order of operators involved, for instance,

$$
\begin{aligned}
& Q_{M}^{N}=(-1)^{M} i^{N} \lim _{x^{ \pm} \rightarrow 0} \frac{\partial^{M}}{\partial\left(x^{-}\right)^{M}} \frac{\partial^{N-M}}{\partial\left(x^{+}\right)^{N-M}} P\left(x^{+}, x^{-}, \tau\right) \\
= & \int_{0}^{\tau} d t_{1} \ldots d t_{N}\left\langle\overleftarrow{T}\left\{A\left(t_{1}\right) \ldots A\left(t_{M}\right)\right\} \vec{T}\left\{A\left(t_{M+1}\right) \ldots A\left(t_{N}\right)\right\}\right\rangle .
\end{aligned}
$$


The moments of (the not necessarily positive) $P(0, q, \tau)$ are expressed through these moments and binomial coefficients,

$$
Q^{(N)} \equiv \int d q q^{N} P(0, q, \tau)=2^{-N} \sum_{M}\left(\begin{array}{l}
N \\
M
\end{array}\right) Q_{M}^{N} .
$$

For $A=I$ these moments correspond to the moments generated by the $\chi(\lambda)$ of the previous section, that is contained in $P\left(x^{+}, x^{-}, \tau\right)$ as $\chi(\lambda)=$ $P(\lambda / 2,-\lambda / 2, \tau)$ [compare Eqs. (5) and (6) in the semiclassical approximation, where $H_{\lambda}=H_{0}-\lambda I$, to Eq. (17)]. Interpreting $\chi(\lambda)$ as the characteristic function corresponding to a probability distribution is therefore equivalent to the classical interpretation of $P(x, q, \tau)$ discussed above. It is applicable to systems with an $x$ - independent $P(x, q, \tau)$.

\subsection{FCS OF A SYSTEM IN THE GROUND STATE}

To acquire a better understanding of the general relations obtained we consider now an important special case. We will assume that the system considered is in its ground state $|g\rangle$, so that its initial density matrix is $R=$ $|g\rangle\langle g|$. In this case the FCS is easily calculated. We have assumed that the coupling between the system and the detector is switched on adiabatically. Then the time development operators in (17) during the time interval $t_{1}<$ $t<0$ adiabatically transfer the system from $|g\rangle$ into the ground state $\left|g\left(x^{ \pm}\right)\right\rangle$of the new Hamiltonian $H_{\text {sys }}-x^{ \pm} A$. In the time interval $0<t<\tau$ the time evolution of the resulting state has then the form

$$
e^{-i t\left(H_{\mathrm{sys}}-x^{ \pm} A\right)}\left|g\left(x^{ \pm}\right)\right\rangle=e^{-i t E\left(x^{ \pm}\right)}\left|g\left(x^{ \pm}\right)\right\rangle .
$$

Here, $E\left(x^{ \pm}\right)$are the energies corresponding to $\left|g\left(x^{ \pm}\right)\right\rangle$. This gives the main contribution to the FCS if the measurement time is large and the phase acquired during the switching of the interaction can be neglected in comparison with this contribution,

$$
P\left(x^{+}, x^{-}, \tau\right)=e^{-i \tau\left[E\left(x^{+}\right)-E\left(x^{-}\right)\right]} .
$$

We now assume the function $E(x)$ to be analytic and expand it in its Taylor series. We also re-scale $q$ as above, $\bar{A}=q / \tau$. We have then for the FCS

$$
P(x, \bar{A}, \tau)=\tau \int d z e^{-i z \bar{A} \tau} \cdot e^{-i \tau\left[E^{\prime}(x) z+E^{\prime \prime \prime}(x) z^{3} / 24+\ldots\right]}
$$

First we observe that $P(x, \bar{A}, \tau)$ is a real function in this case, since the exponent in (30) is anti-symmetric in $z$. A first requirement for being able to interpret it as a probability distribution is therefore fulfilled. However, the 
same asymmetry assures that all even cumulative moments of $\bar{A}$ are identically zero, whereas the odd ones need not. On one hand, since the second moment corresponds to the noise and the ground state cannot provide any, this makes sense. On the other hand, this would be impossible if $P(0, \bar{A}, \tau)$ were a positive probability distribution unless it had no dispersion at all.

Belzig and Nazarov [6] encountered this situation analyzing the FCS of a super-conducting junction. In a certain limit the junction becomes a Josephson junction in its ground state. In this limit the interpretation of the FCS as a probability distribution does not work any longer. Fortunately enough, relation (20) allows us to interpret the results obtained.

In the limit $\tau \rightarrow \infty$ of Eq. (30) all the terms involving higher derivatives of $E(x)$ are negligible and we have

$$
\lim _{T \rightarrow \infty} P(x, \bar{A}, \tau)=\delta\left[\bar{A}+E^{\prime}(x)\right] .
$$

According to the Hellman-Feynman theorem $E^{\prime}(x)=-\langle g(x)|A| g(x)\rangle$. As one would expect, in this limit the measurement gives the expectation value of the operator $A$ in a ground state of the system that is somewhat altered by its interaction with the detector at position $x$. Therefore the resulting dispersion of $A$ will be determined by the initial quantum mechanical spread of the detector wave function. The error of the measurement stems from the interaction with the detector rather than from noise intrinsic in the measured system.

\subsection{FCS OF ELECTRIC CURRENT IN A NORMAL CONDUCTOR}

A complementary example is a normal conductor biased at finite voltage. This is a stationary non-equilibrium system far from being in its ground state. Here we do not go to microscopic details of the derivation. Our immediate aim is to make contact with the approaches of Refs. [2, 5]. We keep the original notations of the references wherever it is possible.

The FCS of the current in a phase-coherent conductor is characterized by a set of transmission coefficients $\Gamma_{n}$ [c. f. Eq. (37) of [2]]. At zero temperature and bias voltage $V$ it reads

$$
P\left(x^{+}, x^{-}, \tau\right)=\exp \left\{\frac{e \tau}{2 \pi}|V| S\left[i e\left(x^{+}-x^{-}\right) \operatorname{sign} V\right]\right\}
$$

with the function

$$
S(\xi)=\sum_{n} \ln \left[1+\left(e^{\xi}-1\right) \Gamma_{n}\right]
$$

This expression depends on $x^{+}-x^{-}$only, as a direct consequence of gauge invariance. Indeed, in every time development operator of Eq. (17) (with 
$A=I$ ) the coupling term is the coupling to a vector potential localized in a certain cross-section of the conductor. The gauge transform that shifts the phase of the wave functions by $e x^{ \pm}$on the right side of that crosssection eliminates this coupling term. This transformation was explicitly implemented in [5]. Since there are two time development operators in the expression, the coupling terms cannot be eliminated simultaneously when $x^{+} \neq x^{-}$. However, the gauge transform with the phase shift $e\left(x^{+}+x^{-}\right) / 2$ makes the coupling terms depending on $x^{+}-x^{-}$only.

Since $P\left(x^{+}, x^{-}, \tau\right)$ depends on $x^{+}-x^{-}$only, the FCS $P(x, q, \tau)$ is independent of $x$. As we have seen in section 3.3, under this condition the FCS can be interpreted as a probability distribution. As in section 2 we conclude that for the statistics of the current of non-interacting electrons the characteristic function $\chi(\lambda)=P(\lambda / 2,-\lambda / 2, \tau)$ is associated with a positive probability distribution.

Superconductivity breaks gauge invariance, thus making such an interpretation impossible.

\section{Electric Circuits as General Linear Detectors}

The model used in the previous section may seem rather abstract and unrealistic. To make contact to the "real world", we notice that

- the time derivative of the detector momentum $q$ is related to the current through the mesoscopic system

- the velocity $\dot{x}$ enters the Hamiltonian in the same way as the voltage applied to the conductor.

Next we adopt the following definition of the "real world": the only quantities measured are electric voltages and currents between nodes of an electric circuit. The most adequate description of the quantum mechanics of the system would thus contain these variables only. This description is hardly possible to achieve within a Hamiltonian formalism, since the latter can contain neither dissipation nor retardation. It is the Feynman-Vernon formalism [4] that allows to formulate quantum mechanics in the form of an action that contains only necessary variables. This action may be derived from the Hamiltonian formalism by tracing out irrelevant degrees of freedom. But this is precisely what we have done in the previous sections! The conclusion is that the above results can be used to arrive at an adequate formulation of the quantum dynamics of electric circuits that contain mesoscopic conductors. This formulation clarifies the notion of a detector.

We formulate the problem as follows. Suppose we know the FCS of a mesoscopic conductor. When measured, it is embedded in an electric circuit. Generally speaking, one does not measure the voltage or current directly at the mesoscopic conductor but rather somewhere else in the cir- 

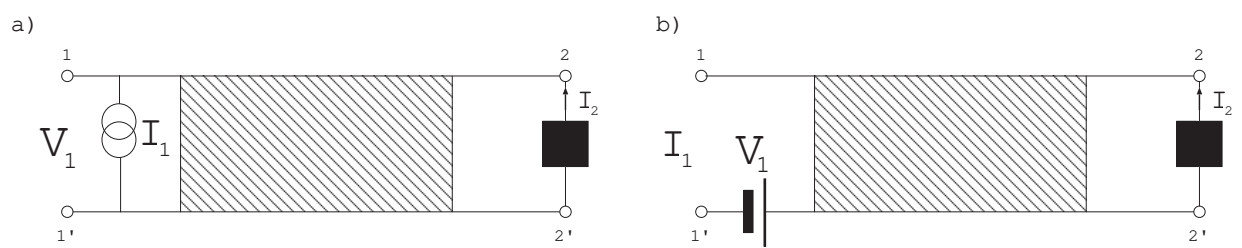

Figure 1. Electric circuit as a general linear detector. The black box symbolizes the electronic system to be measured. It is embedded in a linear environment (shaded box). Either voltage (a) or current (b) is measured between the output contacts 1 and $1^{\prime}$ of the circuit.

cuit. We cast this in Fig. 1 in circuit theory terms. Either a voltage or a current measurement is performed at the output contacts 1 and $1^{\prime}$. The mesoscopic conductor (black box) is connected to the input contacts 2 and $2^{\prime}$. The shaded box presents the electromagnetic environment of the mesoscopic conductor. It is supposed to be a piece of a linear circuit and thus can be characterized separately from the "black box" by three (frequencydependent) response functions. The question is what is the FCS of such a measurement.

We answer it by extending our simple detection model of the previous section to a set of dynamical detectors. We model the "environment", including the detectors, by a set of additional degrees of freedom. This way we study classical back action effects of the detectors. These effects will be more important than the subtle "quantum" influence of the detector on the measured system discussed in the previous section. The detectors, representing the environment, shall obey linear equations of motion. This restriction to linear circuits is compatible with most experimental situations. Detector non-linearities are avoided in many experiments because of undesired effects like the mixing of different frequencies.

\subsection{THE MODEL OF A LINEAR ELECTROMAGNETIC ENVIRONMENT}

Linear systems can be modeled by a set of non-interacting bosons [11]. The Hamiltonian reads

$$
H_{\text {linear }}=\sum_{m} \Omega_{m} a_{m}^{\dagger} a_{m}-\sum_{i} h_{i}(t) H_{i}
$$

where the operators $H_{i}$ are the physical quantities of interest and conjugated to generalized forces $h_{i}$. These operators are linear combinations of $a_{m}^{\dagger}, a_{m}$

$$
H_{i}=\sum_{m} c_{m}^{(i)} a_{m}+h . c . .
$$


A proper choice of $\Omega_{m}, c_{m}^{(i)}$ models arbitrary response functions and enables one to formulate the quantum dynamics of the circuit in terms of quantities of interest only [11]. We are dealing with two pairs of conjugated variables, $\left(\chi_{1}, I_{1}\right)$ and $\left(\chi_{2}, I_{2}\right)$, where $\chi_{j}(t)=\int^{t} d \tau V_{j}(\tau)$. One variable of each pair can be chosen as generalized force and the remaining one as operator. Although different choices lead to different Hamiltonians, the concrete choice is just a matter of convenience. Our primary choice is to treat $I_{2}$ and $\chi_{1}$ as generalized forces. The response functions relate $I_{1}$ and $V_{2}$ to those two variables:

$$
\begin{aligned}
I_{1}(\omega) & =\tilde{Y}(\omega) V_{1}(\omega)+K(\omega) I_{2}(\omega) \\
V_{2}(\omega) & =\tilde{Z}(\omega) I_{2}(\omega)-K(\omega) V_{1}(\omega) .
\end{aligned}
$$

More symmetric choices express either currents in terms of voltages via admittances $Y_{1}, Y_{2}, Y_{12}$,

$$
\begin{aligned}
& I_{1}(\omega)=Y_{1}(\omega) V_{1}(\omega)+Y_{12}(\omega) V_{2}(\omega) \\
& I_{2}(\omega)=Y_{2}(\omega) V_{2}(\omega)+Y_{12}(\omega) V_{1}(\omega)
\end{aligned}
$$

or voltages in terms of currents via impedances $Z_{1}, Z_{2}, Z_{12}$,

$$
\begin{aligned}
& V_{1}(\omega)=Z_{1}(\omega) I_{1}(\omega)+Z_{12}(\omega) I_{2}(\omega) \\
& V_{2}(\omega)=Z_{2}(\omega) I_{2}(\omega)+Z_{12}(\omega) I_{1}(\omega) .
\end{aligned}
$$

There are obvious relations between these response coefficients: $\tilde{Z}=1 / Y_{2}, \tilde{Y}=$ $\left(Y_{1} Y_{2}-Y_{12}^{2}\right) / Y_{2}, K=Y_{12} / Y_{2},\left(Z_{1}, Z_{2}, Z_{12}\right)=\left(Y_{2}, Y_{1},-Y_{12}\right) /\left(Y_{1} Y_{2}-Y_{12}^{2}\right)$.

Here we assume a passive circuit in thermal equilibrium. The response functions then satisfy Onsager symmetry relations and the fluctuationdissipation theorem relates the response functions to fluctuations of the corresponding variables.

\subsection{GENERAL RELATION}

We derive now the general relation that determines the full counting statistics of the output $I_{1}(t)$ of the linear detector described above provided it is coupled to the "black box" to be measured. We will follow the lines of section 3.2. However, now we assume the detector to be in a state of thermal equilibrium at temperature $T$. In addition, instead of evaluating the final density matrix of the detector $\rho^{f}$, we analyze the partition functional $\mathcal{Z}_{I}$ that generates moments of the read-off environment variable $I_{1}$. We define this functional as

$$
\mathcal{Z}_{I}\left[\vec{\chi}_{1}\right]=\left\langle\overleftarrow{\mathrm{T}} e^{i \int d t\left[H_{I}+\chi_{1}^{-}(t) I_{1}\right]} \overrightarrow{\mathrm{T}} e^{-i \int d t\left[H_{I}+\chi_{1}^{+}(t) I_{1}\right]}\right\rangle
$$


It generates moments of outcomes of measurements of $I_{1}(t)$ at any instant of time.

It is advantageous to write the functional in its dependence on the combinations $V_{1}^{c l}=(\partial / \partial t)\left(\chi_{1}^{+}+\chi_{1}^{-}\right) / 2$ and $\chi_{1}^{q}=\left(\chi_{1}^{+}-\chi_{1}^{-}\right) / 2$, that we collect into a "vector" $\vec{\chi}_{1}=\left(V_{1}^{c l}, \chi_{1}^{q}\right)$. The "classical" field $V_{1}^{c l}$ accounts for a (time-dependent) voltage source between the contacts 1 and $1^{\prime}$. Moments of $I_{1}(t)$ are generated by differentiation of $\mathcal{Z}_{I}$ with respect to the antisymmetric, sometimes called "quantum", field:

$$
\left\langle\left[I_{1}\left(t_{1}\right) \ldots I_{1}\left(t_{m}\right)\right]\right\rangle=\left.\frac{\delta}{-2 i \delta \chi_{1}^{q}\left(t_{1}\right)} \ldots \frac{\delta}{-2 i \delta \chi_{1}^{q}\left(t_{m}\right)} \mathcal{Z}_{I}\left[\vec{\chi}_{1}\right]\right|_{\chi^{q}{ }_{1}=0} .
$$

In general, these moments depend on $V_{1}^{c l}$.

$H_{I}$ is the Hamiltonian of the circuit for a current measurement. It reads

$$
H_{I}=H_{\text {linear }}+H_{\text {Sys }}-\chi_{2} I .
$$

$H_{\text {linear }}$ governs the bosonic detector degrees of freedom and $H_{\text {Sys }}$ acts on the electronic degrees of freedom of the "black box". The third term couples the electric current to the detector degree of freedom $\chi_{2}(t)=\int^{t} d t^{\prime} V_{2}\left(t^{\prime}\right)$. The latter is thus the analogue of $x$ in section 3.

Both $\chi_{2}$ and $I_{1}$ are linear combinations of boson creation/annihilation operators,

$$
\chi_{2}=\sum_{m} c_{m}^{(\chi)} a_{m}+c_{m}^{(\chi) *} a_{m}^{\dagger}, \quad I_{1}=\sum_{m} c_{m}^{(I)} a_{m}+c_{m}^{(I) *} a_{m}^{\dagger} .
$$

We rewrite now $\mathcal{Z}_{I}$ as a path integral in detector variables, like we have done to derive Eq. (11). The integration variables now are $a_{m}^{( \pm)}(t), \pm$ corresponding to the two parts of the Keldysh contour. Operators $\chi_{2}, I_{1}$ are replaced by

$$
\chi_{2}^{( \pm)}=\sum_{m} c_{m}^{(\chi)} a_{m}^{( \pm)}+\text {h.c. } I_{1}^{( \pm)}=\sum_{m} c_{m}^{(I)} a_{m}^{( \pm)}+\text {h.c. },
$$

the sign depending on the part of the contour they reside on. To proceed, we introduce two extra vector variables into the path integral: $\vec{\chi}_{2}=\left(V_{2}^{c l}, \chi_{2}^{q}\right)$ and $\vec{q}_{2}=\left(I_{2}^{c l}, q_{2}^{q}\right)$, by inserting the identity

$$
\begin{aligned}
1 & \simeq \int \mathcal{D}\left[V_{2}^{c l}\right] \mathcal{D}\left[\chi_{2}^{q}\right] \prod_{t} \delta\left(2 V_{2}^{c l}-\dot{\chi}_{2}^{+}-\dot{\chi}_{2}^{-}\right) \delta\left(2 \chi_{2}^{q}-\chi_{2}^{+}+\chi_{2}^{-}\right) \\
\simeq & \int \mathcal{D}\left[V_{2}^{c l}\right] \mathcal{D}\left[\chi_{2}^{q}\right] \mathcal{D}\left[I_{2}^{c l}\right] \mathcal{D}\left[q_{2}^{q}\right] \\
& \quad \exp \left\{i \int d t\left[I_{2}^{c l}\left(2 \chi_{2}^{q}-\chi_{2}^{+}+\chi_{2}^{-}\right)-q_{2}^{q}\left(2 V_{2}^{c l}-\dot{\chi}_{2}^{+}-\dot{\chi}_{2}^{-}\right)\right]\right\} .
\end{aligned}
$$


This allows us to split the integrand into two factors. One factor is a trace over "black box" variables. It depends on $\vec{\chi}_{2}(t)$ only and is in fact the influence functional $\mathcal{Z}_{\text {Sys }}$ introduced in section 3.2, Eq. (15) (with $A=I$ ). It generalizes our definition of the FCS of the "black box" biased by the voltage $V^{c l}(t)$ to time dependent arguments. The other factor is a quadratic form in the $a_{m}(t)$. Its Gaussian character enables us to perform the integrations over the $a_{m}$, yielding

$$
\begin{aligned}
\mathcal{Z}_{I}\left[\vec{\chi}_{1}\right] & =\int \mathcal{D}\left[\overrightarrow{\chi_{2}}\right] \mathcal{D}\left[\vec{q}_{2}\right] e^{-i\left\{\mathcal{S}_{\text {env }}\left(\left[\vec{\chi}_{1}\right],\left[\overrightarrow{q_{2}}\right]\right)+\mathcal{S}_{\text {coup }}\left(\left[\overrightarrow{q_{2}}\right],\left[\overrightarrow{\chi_{2}}\right]\right)\right\}} \mathcal{Z}_{\text {Sys }}\left[\vec{\chi}_{2}\right] \\
\mathcal{S}_{\text {env }} & =\int \frac{d \omega}{2 \pi}\left(\overrightarrow{\chi_{1}} \tilde{\tilde{Y}} \overrightarrow{\chi_{1}}+2 \overrightarrow{\chi_{1}} \check{K} \overrightarrow{q_{2}}+\overrightarrow{q_{2}} \tilde{\tilde{Z}} \overrightarrow{q_{2}}\right) \\
\mathcal{S}_{\text {coup }} & =2 \int d t\left[q_{2}^{q}(t) V_{2}^{c l}(t)-I_{2}^{c l}(t) \chi_{2}^{q}(t)\right] .
\end{aligned}
$$

The environmental part of the action is expressed in terms of response functions of our primary choice. The $2 \times 2$ matrices $\check{Y}, \check{K}$ in the time domain are integral kernels depending on the time difference only. In frequency representation they read

$$
\begin{aligned}
& \check{\tilde{Y}}(\omega)=\left(\begin{array}{cc}
0 & \tilde{Y}^{*}(\omega) \\
\tilde{Y}(\omega) & -2 i \omega \operatorname{coth}\left(\frac{\omega}{2 T}\right) \operatorname{Re} \tilde{Y}(\omega)
\end{array}\right), \\
& \check{K}(\omega)=\left(\begin{array}{cc}
0 & -K^{*}(\omega) \\
K(\omega) & 2 \omega \operatorname{coth}\left(\frac{\omega}{2 T}\right) \operatorname{Im} K(\omega)
\end{array}\right) .
\end{aligned}
$$

The matrix $\check{\tilde{Z}}$ is of the same form as $\tilde{\tilde{Y}}$. We can further simplify this relation by integrating over $\overrightarrow{q_{2}}$. The result acquires the more symmetric form

$$
\begin{aligned}
\mathcal{Z}_{I}\left[\vec{\chi}_{1}\right] & =\int \mathcal{D}\left[\overrightarrow{\chi_{2}}\right] e^{-i \mathcal{S}_{\mathrm{env}}\left(\left[\vec{\chi}_{1}\right],\left[\vec{\chi}_{2}\right]\right)} \mathcal{Z}_{\mathrm{Sys}}\left[\vec{\chi}_{2}\right], \\
\mathcal{S}_{\mathrm{env}} & =\int \frac{d \omega}{2 \pi}\left[\overrightarrow{\chi_{1}} \check{Y_{1}} \overrightarrow{\chi_{1}}+2 \overrightarrow{\chi_{1}} \breve{Y_{12}} \vec{\chi}_{2}+\vec{\chi}_{2} \check{Y}_{2} \vec{\chi}_{2}\right],
\end{aligned}
$$

where the matrices $\hat{Y}_{1}, \hat{Y}_{2}, \hat{Y}_{12}$ are defined as in Eq. (48). This is the desired general relation: it expresses the FCS of electric currents through a mesoscopic conductor that is embedded in a linear electric circuit. It is important that all information about the mesoscopic conductor enters through its FCS at voltage bias, $\mathcal{Z}_{\text {Sys }}$.

Let us discuss the relation in some detail. First, let us replace the general four-pole electric circuit by a single two-pole resistor $Z(\omega)$ (see Fig. 2). We do this by choosing $Y_{1}=Y_{2}=-Y_{12}=Y=1 / Z$. The resistor and the 


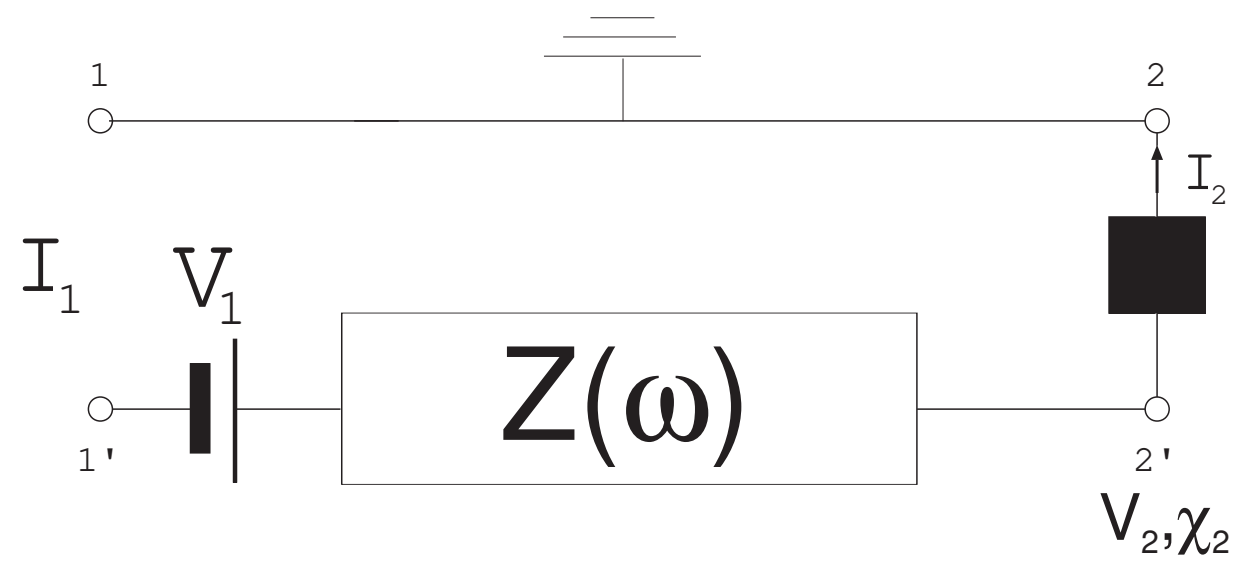

Figure 2. A simple example of a linear environment: a resistor $Z(\omega)$ in series with the "black box". The circuit is biased with a voltage source $V$. The Quantum dynamics of the system is formulated in terms of fluctuating fields $V_{2}, \chi_{2}$.

"black box" enter the expression then on equal footing,

$$
\begin{aligned}
\mathcal{Z}_{I}\left[\vec{\chi}_{1}\right] & =\int \mathcal{D}\left[\vec{\chi}_{2}\right] \mathcal{Z}_{\text {Resistor }}\left[\vec{\chi}_{2}-\vec{\chi}_{1}\right] \mathcal{Z}_{\text {Sys }}\left[\vec{\chi}_{2}\right], \\
\mathcal{Z}_{\text {Resistor }}[\vec{\chi}] & =\exp \left\{-i \int \frac{d \omega}{2 \pi} \vec{\chi} \check{Y} \vec{\chi}\right\}
\end{aligned}
$$

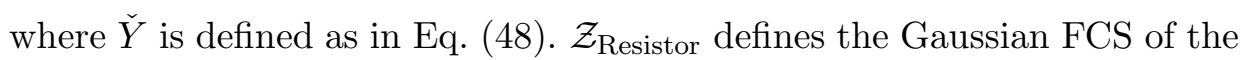
linear voltage-biased two-pole resistor. Eq. (52) expresses a simple concatenation rule for the FCS of compound circuits: the total FCS is a convolution of the FCS's of the individual elements. The resulting FCS is presented as an integral over the fluctuating phase $\vec{\chi}_{2}$ defined in one node of the circuit. This is in agreement with known results about Keldysh actions of electric circuits with Josephson junctions [12]. Moreover, the rule can be easily generalized to more complicated circuits, for example, circuits with two mesoscopic conductors in series.

It is interesting to note that the integration over the fluctuating phase in fact implements a constraint, namely current conservation, on the quantum motion of the compound system. The current through the "black box" equals the input current of the detector:

$$
\begin{aligned}
& \delta\left(I_{\text {Sys }}-I_{1}\right) \rightarrow \prod_{t} \delta\left(I_{\text {Sys }}(t)-I_{1}^{+}(t)\right) \prod_{t} \delta\left(I_{\text {Sys }}(t)-I_{1}^{-}(t)\right) \simeq \\
& \int \mathcal{D} \vec{\chi}_{2} \exp \left\{i \int d t\left[\chi_{2}^{+}(t)\left(I_{\text {Sys }}(t)-I_{1}^{+}(t)\right)-\chi_{2}^{-}(t)\left(I_{\text {Sys }}(t)-I_{1}^{-}(t)\right)\right]\right\},
\end{aligned}
$$


the last equality holding for operators. Since the two currents are represented by operators of a different nature (fermionic for $I_{\text {Sys }}$ and bosonic for $I_{1}$ ), the constraint would be difficult to handle within a Hamiltonian formulation. This illustrates the usefulness of the Keldysh action approach in this context.

So far we have disregarded the constant factors that arise on various stages of the derivation from the variable changes in the path integrals and/or subsequent Gaussian integrations. This is not a matter of our carelessness but rather indicates a problem with the normalization in the approach in use. The normalization of the final relation (50) is known from the fact that $Z_{I}(\vec{\chi})=1$ at $\chi^{q}=0$ per definition of the generating functional. In the path integral representation the correct normalization is assured by the causality structure of the action and integrals over the whole range of frequencies contribute [13]. One can not reproduce the correct factors within the low-frequency approximations and the saddle-point treatment

of the path integrals employed in this article. The problem is not related to the embedding of the mesoscopic conductor and persists also to compound linear circuits. One may draw analogies with known, general and long-standing problems related to the quantum mechanics of constrained motion [14]. To our knowledge it is always possible to correct for wrong factors that arise in calculations by "normalizing" the results to $Z_{I}(\vec{\chi})=1$ at $\chi^{q}=0$. For all general relations given in this article we assume that the correct factor is included into the definition of the metrics in function space, that is, into the definition of $\mathcal{D}\left[\overrightarrow{\chi_{2}}\right]$.

\subsection{VOLTAGE MEASUREMENT AND PSEUDO-PROBABILITIES}

The evaluation of the FCS of electric current through a mesoscopic conductor with voltage bias is a rather straightforward task. One proceeds within the Hamiltonian formulation in terms of electronic operators subjected to an external voltage. The conjugated problem is the FCS of voltage fluctuations under the condition of current bias. The absence of an obvious Hamiltonian formulation for current bias makes this problem less straightforward.

Albeit the problem can be solved in a general way within the approach of the previous subsection. Let us consider the generating functional of voltage fluctuations between 1 and 1' (Fig. 1 b) defined by

$$
\mathcal{Z}_{V}\left[\vec{q}_{1}\right]=\left\langle\overleftarrow{\mathrm{T}} e^{i \int d t\left[H_{V}+q_{1}^{-}(t) V_{1}\right]} \overrightarrow{\mathrm{T}} e^{-i \int d t\left[H_{V}+q_{1}^{+}(t) V_{1}\right]}\right\rangle
$$

Similarly to Eq. (40), derivatives of this functional with respect to $q^{q}(t)$ give the moments of voltage fluctuations. We now repeat the derivation of 
the previous subsection for this functional. The answer reads:

$$
\begin{aligned}
\mathcal{Z}_{V}\left[\vec{q}_{1}\right] & =\int \mathcal{D}\left[\vec{q}_{2}\right] \mathcal{D}\left[\vec{\chi}_{2}\right] e^{-i\left\{\mathcal{S}_{\text {env }}\left(\left[\vec{q}_{1}\right],\left[\vec{q}_{2}\right]\right)+\mathcal{S}_{\text {coup }}\left(\left[\overrightarrow{q_{2}}\right],\left[\overrightarrow{\chi_{2}}\right]\right)\right\}} \mathcal{Z}_{\text {Sys }}\left[\vec{\chi}_{2}\right] \\
\mathcal{S}_{\text {env }} & =\int \frac{d \omega}{2 \pi}\left[\overrightarrow{q_{1}} \check{Z}_{1} \overrightarrow{q_{1}}+2 \overrightarrow{q_{1}} \check{Z}_{12} \vec{q}_{2}+\vec{q}_{2} \check{Z}_{2} \vec{q}_{2}\right] \\
\mathcal{S}_{\text {coup }} & =2 \int d t\left[q_{2}^{q}(t) V_{2}^{c l}(t)-I_{2}^{c l}(t) \chi_{2}^{q}(t)\right]
\end{aligned}
$$

The matrices $\check{Z}_{1,2,12}(\omega)$ are defined analogously to (48) with the impedances (38).

We now choose the linear detector in such a way that the voltage fluctuations between 1 and 1' are the same as between 2 and 2' and the conditions of current bias are met. Elementary circuit theory tells us that this is achieved in the limit $Z_{12} \rightarrow-Z_{1}=-Z_{2} \rightarrow-\infty$. In the expression (55) this corresponds to setting $\vec{q}_{1}=\vec{q}_{2}$ and $\mathcal{S}_{\text {env }}=0$.

This brings us to a remarkable conclusion: for any conductor, the generating functionals for voltage noise at current bias $I^{c l}(t)$ and for current noise at voltage bias $V^{c l}(t)$ are related by a functional Fourier transform,

$$
\mathcal{Z}_{V}[\vec{q}]=\int \mathcal{D}[\vec{\chi}] e^{-2 i \int d t\left(q^{q} V^{c l}-I^{c l} \chi^{q}\right)} \mathcal{Z}_{I}[\vec{\chi}] .
$$

Therefore, the functionals $\mathcal{Z}_{V}$ and $\mathcal{Z}_{I}$ are in fact just different forms of the same object. There is some uncertainty in this definition stemming from the problem mentioned in the previous section. $\mathcal{Z}_{V}$ has to be "normalized" such that $\mathcal{Z}_{V}=1$ at $q^{q}(t)=0$. We assume the normalization factor to be included into the definition of $\mathcal{D}$.

This simple relation between $\mathcal{Z}_{V}$ and $\mathcal{Z}_{I}$ suggests to define the functional

$$
\begin{aligned}
\tilde{P}([V],[I]) & =\int \mathcal{D}\left[q^{q}\right] e^{2 i \int d t q^{q} V} \mathcal{Z}_{V}\left[\left(\begin{array}{c}
I \\
q^{q}
\end{array}\right)\right] \\
& =\int \mathcal{D}\left[\chi^{q}\right] e^{2 i \int d t \chi^{q} I} \mathcal{Z}_{I}\left[\left(\begin{array}{c}
V \\
\chi^{q}
\end{array}\right)\right]
\end{aligned}
$$

that depends on "classical" variables only. We show with the help of Eq. (40) that $\tilde{P}$ has the following properties:

$$
\left.\left\langle V\left(t_{1}\right) \ldots V\left(t_{m}\right)\right\rangle\right|_{I(t)}=\frac{\int \mathcal{D}[V] V\left(t_{1}\right) \ldots V\left(t_{m}\right) \tilde{P}([V],[I])}{\int \mathcal{D}[V] \tilde{P}([V],[I])},
$$

and

$$
\left.\left\langle I\left(t_{1}\right) \ldots I\left(t_{m}\right)\right\rangle\right|_{V(t)}=\frac{\int \mathcal{D}[I] I\left(t_{1}\right) \ldots I\left(t_{m}\right) \tilde{P}([V],[I])}{\int \mathcal{D}[I] \tilde{P}([V],[I])}
$$


Eqs. (60) and (61) resemble the properties of a probability density and yet $\tilde{P}$ is not a probability. First, it does not have to be positive, just as the $P(x, q, \tau)$ discussed in section 3 needs not. Second, $\tilde{P}$ is dimensionless in contrast to a true probability density of either voltage or current fluctuations. For these reasons we refer to $\tilde{P}$ as a 'pseudo-probability'.

$\tilde{P}([V],[I])$ is a characteristics of a two-pole conductor and takes a simple form for a linear circuit. In this case $\tilde{P}$ is positive and depends only on the impedance of the conductor $Z(\omega)$ and its temperature $T$,

$$
\tilde{P}_{Z}([V],[I])=\exp \left\{-\int \frac{d \omega}{4 \pi} \frac{|V(\omega)-Z(\omega) I(\omega)|^{2}}{\omega \operatorname{Re} Z} \tanh \frac{\omega}{2 T}\right\} .
$$

From Eq. (60) one derives the voltage correlator

$$
\left\langle V(t)^{2}\right\rangle=\int \frac{d \omega}{2 \pi} \omega \operatorname{Re} Z(\omega) \operatorname{coth} \frac{\omega}{2 T},
$$

that conforms to the fluctuation-dissipation theorem.

The general relation (50) can be rewritten in terms of pseudo-probabilities. In this case, it will express the pseudo-probability of current/voltage fluctuations between 1 and 1 ' $\tilde{P}_{1}([V],[I])$ in terms of the $\tilde{P}_{\text {Sys }}$ of the "black box" and a four-pole pseudo-probability $\tilde{P}_{12}$ that depends on two currents and voltages, characterizing the linear part of the circuit:

$$
\tilde{P}_{1}\left(\left[V_{1}\right],\left[I_{1}\right]\right)=\int \mathcal{D}\left[V_{2}\right] \mathcal{D}\left[I_{2}\right] \tilde{P}_{12}\left(\left[V_{1}\right],\left[I_{1}\right] ;\left[V_{2}\right],\left[I_{2}\right]\right) \tilde{P}_{\mathrm{Sys}}\left(\left[V_{2}\right],\left[I_{2}\right]\right)
$$

We cast now also the result Eq. (52) for the "black box" in series with a linear resistor $Z(\omega)$ in terms of pseudo-probabilities. The answer is expressed in terms of the pseudo-probability of the resistor defined by Eq. (62):

$$
\tilde{P}_{1}([V],[I])=\int \mathcal{D}\left[V_{2}\right] \tilde{P}_{Z}\left(\left[V-V_{2}\right],[I]\right) \tilde{P}_{\text {Sys }}\left(\left[V_{2}\right],[I]\right) .
$$

The above relations are transparent if one interprets them in terms of classical probabilities. They show that the probability of a certain current/voltage fluctuation is composed of a probability of fluctuations in the "black box" and of a probability of fluctuations in the linear detector, voltage and current satisfying circuit theory rules. Yet the relations are quantum mechanical ones and are written for pseudo-probabilities. They contain all necessary information about quantum properties of the system under consideration.

To summarize, fluctuations in a mesoscopic system that is embedded in a linear environment can be characterized by three statements. First, 
voltages and currents are related by circuit theory rules. For instance, for the circuit of Fig. 2 the current fluctuation $\delta I$ in the "black box" results in the current fluctuation $\delta I /(1+Z G)$ in the whole circuit, $G$ being the linear conductance of the "black box". This factor is rather trivial from a classical point of view. However, in the context of quantum mechanical detection, it constitutes the main source of detector back-action. This "classical" backaction could be disregarded for the static detector considered in section 3 . Second, the linear part of the circuit produces its own Gaussian quantum and thermal fluctuations. Also these fluctuations contribute to the FCS of the compound system. Third, the fluctuations in the linear environment affect the fluctuations produced by the "black box". This effect is the least trivial and we will explore its consequences in section 6 .

\section{Low-frequency limit}

We have shown in the previous section that the FCS of a mesoscopic conductor in a general linear environment can be expressed by the relation (50). This relation involves path integration and defines a quantum field theory with the field $\vec{\chi}_{2}(t)$. This field theory is non-linear, the non-linearities coming from the mesoscopic conductor. The latter is sometimes underestimated, since the $I-V$ curve of the conductor may be perfectly linear. However, even in this case the noise and higher order correlators in general do depend on the voltage, which provides the non-linearity.

Such non-linearities make the general problem already hardly tractable. To complicate the situation further, the most important part of the action, $\ln \mathcal{Z}_{\text {Sys }}$, is only known for stationary $\vec{\chi}$. In this case it is given by the relation (32). Microscopic Keldysh Green function techniques can be used to evaluate this functional at non-stationary $\vec{\chi}$. The answer has, however, a complicated functional dependence on the argument $\vec{\chi}$.

Fortunately enough, there is a simple low-frequency limit where the quantum field theory becomes tractable. This limit applies to many experimental situations. In this limit we consider only quasi-stationary realizations of the fields. The action for these realizations is local in time, which allows for easy path integration.

Before turning to the concrete formulation, let us discuss the time scales that occur in our problem and that may determine the non-locality of the action. There may be a time scale, the $R C$-time, characterizing the frequency dependence of the detector response functions $Y_{1,2,12}$. This scale depends on the system layout and can be easily changed. From the experimental point of view it is convenient to choose the response functions to be constant in a wide frequency interval. This allows us to disregard this

time scale. Another time scale, $\tau_{Q}$, has quantum mechanical origin. The 
environment action (51) becomes non-local at frequencies $\omega \simeq k_{B} T$, where the crossover from classical to quantum noise occurs. For the mesoscopic conductor a similar scale may arise from the energy $e V$ related to single electron transfer through the conductor. Therefore $\tau_{Q} \simeq \hbar / \max \left\{e V, k_{B} T\right\}$. A third time scale is the typical time interval $\tau_{I}$ between electron transfers through the mesoscopic conductor, $e / I$. Comparing the latter two scales, we find that $t_{I} \simeq\left(e^{2} / h G\right) \tau_{Q}, G$ being the conductance of the "black box". Generally, we expect the low-frequency limit to be valid at time scales exceeding both $\tau_{I}$ and $\tau_{Q}$. These time scales are typically short in comparison with the time resolution of the measurement electronics. Consequently most electric measurements are performed in the low-frequency limit.

In this limit, the general relation (50) takes the following form:

$$
\begin{array}{r}
Z_{I}\left(\left[V_{1}\right],\left[\chi_{1}\right]\right)=\int \mathcal{D}\left[V_{2}\right] \mathcal{D}\left[\chi_{2}\right] \exp \left\{-\int d t\left[\mathcal{E}_{\text {env }}\left(V_{1}(t), \chi_{1}(t) ; V_{2}(t), \chi_{2}(t)\right)\right.\right. \\
\left.\left.+\mathcal{E}_{\text {Sys }}\left(V_{2}(t), \chi_{2}(t)\right)\right]\right\} ; \quad(66)
\end{array}
$$

where

$$
\begin{aligned}
\mathcal{E}_{\mathrm{env}}\left(V_{1}, \chi_{1} ; V_{2}, \chi_{2}\right)= & -2 i\left[\left(V_{1} Y_{1}+V_{2} Y_{12}\right) \chi_{1}+\left(V_{2} Y_{2}+V_{1} Y_{12}\right) \chi_{2}\right] \\
& +4 T\left(Y_{1} \chi_{1}^{2}+Y_{2} \chi_{2}^{2}+2 Y_{12} \chi_{1} \chi_{2}\right), \\
\mathcal{E}_{\mathrm{Sys}}= & -\ln Z_{\mathrm{Sys}}(V, \chi) / \tau .
\end{aligned}
$$

In the last equation, $Z_{\text {Sys }}$ is evaluated for stationary $V, \chi$. Thus its logarithm is proportional to the measurement time $\tau$ (see Eq. (32)). For the resistor in series (Fig. 2) $\mathcal{E}_{\text {env }}$ reduces to:

$$
\mathcal{E}_{\text {env }}=\mathcal{E}_{Z}\left(V_{1}-V_{2}, \chi_{1}-\chi_{2}\right) ; \mathcal{E}_{Z}(V, \chi)=(-2 i V+4 T \chi) \chi / Z .
$$

As expected in the low-frequency limit, the noise of the detector is just Johnson-Nyquist white noise.

A simple estimation shows that the action is not only local in time. It is also big for the time intervals in question. This allows us to implement a semiclassical approximation, that is, to evaluate the path integrals in saddle-point approximation. Indeed, the typical values of $\chi$, manifesting the discreteness of charge, are of the order of $1 / e$. Typical values of the action are then estimated as $S \simeq I \chi \tau \simeq I / e \tau \simeq\left(\tau / \tau_{I}\right)$. By definition of the low-frequency limit, $\tau / \tau_{I} \gg 1$.

We now come back to the central problem addressed in this article: the distribution of currents averaged over the time interval $\tau$. Taking the path integrals in saddle-point approximation, we arrive at a simple expression for the FCS of the "black box" embedded in an environment circuit:

$$
\begin{aligned}
Z_{I}\left(V_{1}, \chi_{1}\right) & =\exp \left[-\mathcal{E}_{I}\left(V_{1}, \chi_{1}\right) \tau\right] \\
\mathcal{E}_{I}\left(V_{1}, \chi_{1}\right) & =\underset{V_{2}, \chi_{2}}{\operatorname{extr}}\left\{\mathcal{E}_{\text {env }}\left(V_{1}, \chi_{1} ; V_{2}, \chi_{2}\right)+\mathcal{E}_{\text {Sys }}\left(V_{2}, \chi_{2}\right)\right\} .
\end{aligned}
$$


The corresponding FCS of voltage fluctuations at given current $I$ can be obtained directly from Eq. (68) by using Eq. (58),

$$
\begin{aligned}
Z_{V}(I, q) & =\exp \left[-\mathcal{E}_{V}(I, q) \tau\right] \\
\mathcal{E}_{V}(I, q) & =\operatorname{extr}_{V, \chi}\left\{\mathcal{E}_{I}(V, \chi)-2 i(I \chi-q V)\right\} .
\end{aligned}
$$

When integrating Eq. (58) we take care of the normalization to assure that $Z_{V}(I, 0)=1$. A similar integration determines the pseudo-probability:

$$
\begin{aligned}
\tilde{P}(I, V) & =\exp (-\mathcal{E}(V, I) \tau), \\
\mathcal{E}(I, V) & =\underset{\chi}{\operatorname{extr}}\left\{\mathcal{E}_{I}(V, \chi)-2 i I \chi\right\} .
\end{aligned}
$$

Finally, we give here expressions for real probabilities to measure the voltage $V$ at a given current $I, P_{V}(I)$, and to measure the current $I$ at a given voltage $V, P_{I}(V)$. By virtue of the relations (60) and (61) we obtain

$$
\left\{\begin{array}{l}
P_{V}(I) \\
P_{I}(V)
\end{array}\right\}=\sqrt{\frac{\pi}{2 \tau}}\left\{\begin{array}{l}
\sqrt{\frac{\partial I^{2}}{\partial^{2} \mathcal{E}}} \\
\sqrt{\frac{\partial V^{2}}{\partial^{2} \mathcal{E}}}
\end{array}\right\} \tilde{P}(I, V) .
$$

Remarkably, these probabilities are in fact the same with exponential accuracy. They differ in normalization factors only,

$$
\frac{P_{V}(I)}{P_{I}(V)}=\sqrt{\frac{\partial I^{2}}{\partial^{2} \mathcal{E}} \frac{\partial^{2} \mathcal{E}}{\partial V^{2}}} .
$$

The relations (69-72) are valid for the statistics of the outputs of the compound circuit as well as for the "black box" biased by either a voltage or a current source.

The FCS in the low-frequency limit can thus be formulated in terms of stationary functions $\mathcal{E}_{I}, \mathcal{E}_{V}, \mathcal{E}(I, V)$. These functions resemble thermodynamic potentials. As those potentials, they are related by Legendre transforms. However, the relations presented concern non-equilibrium systems. They provide an effective minimum principle for fluctuations in such systems.

\subsection{WHERE ARE THE CHARGING EFFECTS?}

The electron transport through mesoscopic circuits is known to be affected by charging or Coulomb blockade effects. They are due to electron-electron interactions at the mesoscopic scale. The simplest case of a tunnel junction embedded into an electromagnetic environment is described in [15] in some

detail. Although these charging effects are not the topic of this article, we 
find it important to note that they are present in our formalism. Indeed, the Keldysh action in use is a generalization of the action introduced in [12] to describe Coulomb blockade effects in Josephson junctions.

How do charging effects manifest themselves in the low-frequency limit considered?

The way to understand this is to invoke the field-theoretical concept of renormalization. We have obtained the low-frequency action just by substituting slow realizations of the fluctuating field $\vec{\chi}_{2}$ into the original action. The renormalization procedure gives a more accurate way to obtain the low-frequency action. In this procedure, the field is separated into a slow and a fast part. The path integration over the fast variables is performed. Since fast and slow variables are coupled, this alters the functional of the slow variables, it is "renormalized". For the problem under consideration, this renormalization introduces charging effects. Since the environment action is quadratic, it is not renormalized. The renormalization can be thus ascribed to $\mathcal{E}_{\text {Sys }}$. The typical time scale of relevant fast modes is $\tau_{Q}$. The importance of the charging effects is governed by the value of the environment impedance at the corresponding frequencies, $Z\left(1 / \tau_{Q}\right)[15]$. If $Z\left(1 / \tau_{Q}\right) \ll \hbar / e^{2}$, the renormalization is small and charging effects can be disregarded. In the opposite case, charging effects are important and the renormalized action $\mathcal{E}$ differs significantly from the bare one.

We thus conclude that charging effects do not change the low-frequency relations given in the previous subsection. Their only effect is a renormalization of $\mathcal{E}$ in comparison to its bare value at voltage bias. This renormalization depends on the environment response functions at the frequency scale $1 / \tau_{Q}$.

\section{Mesoscopic conductor in a macroscopic circuit}

In this section, based on Ref. [9], we illustrate the general relations obtained above. We do this by considering a specific example: a phase-coherent conductor in series with an impedance $Z$ (Fig. 2) [9]. Further we focus on the shot noise limit $e V \gg k_{B} T$, and disregard the Johnson-Nyquist noise produced by the linear resistor. First we address the FCS of current fluctuations in the low-frequency limit discussed above. We will thus make use of the relations given in section 5. The assumption $Z(\omega \simeq e V) \ll \hbar / e^{2}$ allows us to disregard charging effects without any restrictions on $Z(0)$.

To employ our scheme, we first need the concrete expression for the FCS of the voltage-biased conductor, $\mathcal{Z}_{\text {Sys }}$. In the stationary case, this has been derived in [2]. The mesoscopic conductor is completely characterized by its transmission eigenvalues $\Gamma_{n}$ [16] that are assumed not to depend on energy. In our notations, we have $\left(|e V| \gg k_{B} T\right)$ 


$$
\mathcal{Z}_{\text {Sys }}(V, \chi)=\exp \left\{-\mathcal{E}_{\text {Sys }} \tau\right\}, \quad \mathcal{E}_{\text {Sys }}=-\frac{e}{2 \pi}|V| S[2 i e \chi \operatorname{sign}(V)]
$$

with the dimensionless $S$ of Eq. (33).

Since we disregard the noise of the resistor, we have the environment action $\mathcal{E}_{\mathrm{Z}}(V, \chi)=-2 i V Z^{-1} \chi$. By virtue of Eq. (68), the FCS of the circuit is defined by

$$
\mathcal{E}_{I}(V, \chi)=\operatorname{extr}_{V_{2}, \chi_{2}}\left\{-2 i Z^{-1}\left(V_{2}-V\right)\left(\chi_{2}-\chi\right)-\frac{e\left|V_{2}\right|}{2 \pi} S\left[2 i e \chi_{2} \operatorname{sign}\left(V_{2}\right)\right]\right\} .
$$

Minimization with respect to $V_{2}$ fixes $\chi_{2}$,

$$
2 i Z^{-1}\left(\chi-\chi_{2}\right)=\frac{e}{2 \pi} S\left(2 i e \chi_{2}\right),
$$

and makes further minimization with respect to this variable redundant. We immediately arrive at

$$
\mathcal{E}_{I}(V, \chi)=-\frac{e V}{2 \pi} S\left[2 i e \chi_{2}(\chi)\right]
$$

where $\chi_{2}(\chi)$ is implicitly defined by Eq. (75). In the limit of a vanishing external impedance $Z \rightarrow 0$, we have $\chi_{2} \rightarrow \chi$ so that $\mathcal{E}_{I}(V, \chi) \rightarrow \mathcal{E}_{\text {Sys }}$, as it should.

The coefficients of a series expansion of $\mathcal{E}_{I}$ in $\chi$ give the cumulants of the transmitted charge $\left\langle\left\langle Q^{p}\right\rangle\right\rangle$, whereas the coefficients of $\mathcal{E}_{\text {Sys }}$ are the same cumulants $\left\langle\left\langle Q^{p}\right\rangle_{0}\right.$ in the limit $Z \rightarrow 0$. Comparing the two series, we obtain relations between these cumulants. The linear terms imply $\langle Q\rangle=$ $(1+Z G)^{-1}\langle Q\rangle_{0}, G$ being the conductance of the mesoscopic conductor. This is a consequence of Ohm's law for the average current $\bar{I}$ that is reduced by a factor $1+Z G$ by the resistor $Z$ in series. The Langevin approach of Ref. [17] predicts the same re-scaling for the fluctuations of the current. Indeed, in second order we find that $\left\langle\left\langle Q^{2}\right\rangle\right\rangle=\left(1+Z_{2} G\right)^{-3}\left\langle\left\langle Q^{2}\right\rangle\right\rangle_{0}$, in agreement with this prediction.

However, for higher order cumulants we find terms that are not consistent with this re-scaling hypothesis. For instance, the third cumulant reads

$$
\left\langle\left\langle Q^{3}\right\rangle\right\rangle=\frac{\left\langle\left\langle Q^{3}\right\rangle\right\rangle_{0}}{(1+Z G)^{4}}-\frac{3 Z G}{(1+Z G)^{5}} \frac{\left(\left\langle\left\langle Q^{2}\right\rangle\right\rangle_{0}\right)^{2}}{\langle Q\rangle_{0}} .
$$

Although the first term on the right-hand-side does have the scaling form conjectured, the second term does not. This is generic for $p \geq 3$ : $\left\langle\left\langle Q^{p}\right\rangle\right\rangle=$ $(1+Z G)^{-p-1}\left\langle\left\langle Q^{p}\right\rangle\right\rangle_{0}$ plus a non-linear (multinomial) function of lower cumulants. This mixing in of lower order cumulants is a consequence of the 
non-linear feedback mechanism discussed in the end of section 4.3. To give another example, the forth cumulant reads

$$
\begin{aligned}
\left\langle\left\langle Q^{4}\right\rangle\right\rangle= & \frac{\left\langle\left\langle Q^{4}\right\rangle\right\rangle_{0}}{\left(1+Z_{2} G\right)^{5}}- \\
& -\frac{10 Z G}{\left(1+Z_{2} G\right)^{6}} \frac{\left\langle\left\langle Q^{2}\right\rangle\right\rangle_{0}\left\langle\left\langle Q^{3}\right\rangle\right\rangle_{0}}{\langle Q\rangle_{0}}+\frac{15 Z^{2} G^{2}}{\left(1+Z_{2} G\right)^{7}} \frac{\left\langle\left\langle Q^{2}\right\rangle\right\rangle_{0}^{3}}{\langle Q\rangle_{0}^{2}} .
\end{aligned}
$$

We now turn to a one-channel conductor with transmission probability $\Gamma$. In this case, the distribution of the integer transmitted charge $q \equiv I \tau e$ for voltage bias $V$ is known to take the simple binomial form [2] ( $\phi \equiv e V \tau / 2 \pi)$

$$
P_{\phi}(q)=\left(\begin{array}{c}
\phi \\
q
\end{array}\right) \Gamma^{q}(1-\Gamma)^{\phi-q}
$$

The distribution dual to this is that of the flux $\phi \equiv(e / h) \int_{0}^{\tau} d t V(t)$ that is accumulated at the conductor under current bias $I$. We make use of to the relation (70) that determines these probabilities. For a mesoscopic conductor in the shot noise limit, the corresponding $\mathcal{E}(I, V)$ reads

$$
\mathcal{E}(I, V)=\underset{\chi}{\operatorname{extr}}\left\{\frac{e V}{2 \pi} S(2 i e \chi)-2 i I \chi\right\}
$$

Expanding this near its point of extremum, we find that

$$
\frac{\partial I^{2}}{\partial^{2} \mathcal{E}} \frac{\partial^{2} \mathcal{E}}{\partial V^{2}}=\frac{I^{2}}{V^{2}}
$$

for any functional form of $S$. Employing Eq. (72) we readily establish that the distribution function of flux $P_{q}(\phi)$ is given by (here we set $q \equiv I \tau / e$ )

$$
P_{q}(\phi)=\frac{q}{\phi} P_{\phi}(q)=\left(\begin{array}{c}
\phi-1 \\
q-1
\end{array}\right) \Gamma^{q}(1-\Gamma)^{\phi-q},
$$

which is known as the Pascal distribution.

We complement this derivation by a simple interpretation of the Pascal distribution (82) of voltage fluctuations. The binomial distribution (79) of transferred charge can be interpreted [2] in gambling terms: it gives the probability to win (transfer an electron) $q$ times in $\phi_{0}$ game slots given a winning chance of $\Gamma$. In the voltage biased conductor, electrons leave the leads with a frequency $\mathrm{eV} / \mathrm{h}$ and $\phi_{0}=(e / h) \int_{0}^{\tau} d t V(t)$ is the number of electrons ("game slots") that try to pass the barrier in the detection time window. 
The current bias changes the rules of the game. The gambling now ends when precisely $q_{0}$ attempts have been successful. This requires a fluctuating number $\phi$ of game slots (electrons that hit the barrier). Indeed, the Pascal distribution quantifies the probability distribution of the number of independent trials that one needs to achieve a given number of successes in a Bernoulli experiment.

\section{References}

1. L. S. Levitov and G. B. Lesovik, JETP Lett. 55, 555 (1992).

2. L. S. Levitov and G. B. Lesovik, JETP Lett. 58, 230 (1993); L. S. Levitov, H. W. Lee, and G. B. Lesovik, Journ. Math. Phys. 37, 4845 (1996).

3. J. Rammer and H. Smith, Rev. Mod. Phys. 58, 323 (1986).

4. R.P. Feynman and F.L. Vernon, Ann. Phys.(N.Y.) 24, 118 (1963).

5. Yu. V. Nazarov, Ann. Phys. (Leipzig) 8 Spec. Issue SI-193, 507 (1999).

6. W. Belzig and Yu. V. Nazarov, Phys. Rev. Lett. 87, 197006 (2001).

7. A. Shelankov and J. Rammer, cond-mat/0207343 (2002).

8. Yu. V. Nazarov and M. Kindermann, cond-mat/0107133 (2001).

9. M. Kindermann, Yu.V. Nazarov, and C.W.J. Beenakker, cond-mat/0210617 (2002).

10. J. v. Neumann, Die mathematischen Grundlagen der Quantenmechanik, Springer Verlag, Berlin (1932).

11. A.O. Caldeira and A.J. Leggett, Phys. Rev. Lett. 46, 211 (1981).

12. G. Schön and A.D. Zaikin, Phys. Rep. 198, 237 (1990).

13. A. Altland and A. Kamenev, Phys. Rev. Lett. 85, 5615 (2000).

14. L. Kaplan, N. T. Maitra and E. J. Heller, Phys. Rev. A 56, 2592 (1997).

15. G.-L. Ingold and Yu. V. Nazarov, in Single Charge Tunneling, edited by H. Grabert and M. H. Devoret, NATO ASI Series B294 (Plenum, New York, 1992).

16. C. W. J. Beenakker, Rev. Mod. Phys. 69, 731 (1997).

17. Ya. M. Blanter and M. Büttiker, Phys. Rep. 336, 1 (2000). The effect of a series resistance on the noise power is discussed in $\S 2.5$. 